\title{
A Step Forward in the Treatment of Influenza
}

\author{
Masoud Mardani (ii) ${ }^{1, *}$ \\ ${ }^{1}$ Infectious Diseases and Tropical Medicine Research Center, Shahid Beheshti University of Medical Sciences, Tehran, Iran \\ "Corresponding author: Infectious Diseases and Tropical Medicine Research Center, Shahid Beheshti University of Medical Sciences, Tehran, Iran. Email: \\ drmasoudmardani@yahoo.com
}

Received 2019 December 07; Accepted 2019 December 07.

Keywords: Influenza, Baloxivir, Treatment

Influenza had a terrible season in the last year, in particular, the seasonal influenza A (H3N2) virus that was estimated by the CDC to have caused 80,000 deaths and the highest number of confirmed pediatric deaths ever known (1). Influenza antiviral therapy has been a monotherapy with a neuraminidase inhibitor for many years. The Food and Drug Administration (FDA) approved oseltamivir (oral administration) and zanamivir (oral inhalation) inhibitors of neuraminidase in 1999 and peramivir (intravenous administration) in late 2014 . These drugs work by binding to the viral neuraminidase protein and inhibiting the release of influenza virus particles from infected respiratory tract cells. Neuraminidase inhibitors are FDAapproved for the treatment of uncomplicated influenza within two days of the onset in outpatients based on randomized controlled trials, but are also recommended for the treatment of influenza patients (2). The CDC has advised anyone who is hospitalized or at risk of serious illness to use the drug, regardless of the timing of the infection. We know that the drug works best if taken at a very early stage (within the first 48 hours), but the data are mixed as to whether it is helpful for patients (3). Oseltamivir is only available as an oral drug. Peramivir, which is available as a single intravenous dose, is occasionally used in very ill patients who are unable to take oseltamivir. Zanamivir has precious little use as an inhaled powder because the patient wants to take the powder when the patient has a respiratory illness (1). Baloxovir is a new oral drug with a very long half-life of almost 80 hours; thus, it is given as a single dose. This drug works differently from oseltamivir; it inhibits acidic polymerase endonuclease and thus interferes with viral replication much earlier in the viral lifecycle (1). Earlier last year, the New England Journal of Medicine published the results of a study comparing baloxavir with placebo and oseltamivir. Drugs were taken within 48 hours of the onset of symptoms. Baloxavir worked reasonably well, as did oseltamivir. Fever decreased by one day; patients felt better one day earlier. There was a rather marked reduction in the viral shedding of baloxavir much better than what was seen with oseltamivir, probably because baloxavir worked earlier in the viral lifecycle (4). Baloxavir appeared to have a favorable side-effect profile compared to placebo. Side effects were fewer with baloxavir than with placebo and oseltamivir. At least in higher-risk patients, baloxavir appeared to decrease the rate of influenza complications and antibiotic use in this population (1).

Patients may take baloxavir as a single dose instead of taking it twice daily for five days (like oseltamivir), which is advantageous. Obviously, baloxavir is a branded drug, so its higher costs must be taken into account. One of the more interesting questions is whether reduced viral shedding associated with baloxavir will be beneficial, as perhaps fewer people will become infected in households or hospitals (1).

Moreover, baloxavir is effective in the treatment of avian influenza and probably it has a major role in the next pandemic. Therefore, a comprehensive review is suggested on how this drug works in avian influenza and epidemic influenza cases. Unfortunately, it is noticeable that Iranian physicians have no access to new anti-influenza drugs while even limited access in severe cases can be very helpful.

\section{Footnotes}

Conflict of Interests: None.

Funding/Support: None declared by the author.

\section{References}

1. Centers for Disease Control and Prevention; National Center for Immunization and Respiratory Diseases (NCIRD). Summary of the 2017- 
2018 influenza season. CDC; 2019. Available from: https://www.cdc. gov/flu/about/season/flu-season-2017-2018.htm.

2. Uyeki TM. A step forward in the treatment of influenza. N Engl J Med. 2018;379(10):975-7. doi: 10.1056/NEJMe1810815. [PubMed: 30184452]. [PubMed Central: PMC6669030].

3. Ramirez J, Peyrani P, Wiemken T, Chaves SS, Fry AM. A randomized study evaluating the effectiveness of oseltamivir initiated at the time of hospital admission in adults hospitalized with influenza-associated lower respiratory tract infections. Clin Infect Dis. 2018;67(5):736-42. doi: 10.1093/cid/ciy163. [PubMed: 29659754].

4. Hayden FG, Sugaya N, Hirotsu N, Lee N, de Jong MD, Hurt AC, et al. Baloxavir marboxil for uncomplicated influenza in adults and adolescents. NEnglJMed. 2018;379(10):913-23. doi: 10.1056/NEJMoa1716197. [PubMed: 30184455]. 